\title{
In silico toxicology models and databases as FDA Critical Path Initiative toolkits
}

Luis G. Valerio, Jr.*

Office of Pharmaceutical Science, Center for Drug Evaluation and Research, US Food and Drug Administration, White Oak 51, Room 4128, 10903 New Hampshire Avenue, Silver Spring, MD 20993-0002, USA.

*Correspondence to: Tel: +1 301796 0208; Fax: +1 301796 9997; E-mail: luis.valerio@fda.hhs.gov

Date received (in revised form): 8th October 2010

\begin{abstract}
In silico toxicology methods are practical, evidence-based and high throughput, with varying accuracy. In silico approaches are of keen interest, not only to scientists in the private sector and to academic researchers worldwide, but also to the public. They are being increasingly evaluated and applied by regulators. Although there are foreseeable beneficial aspects - including maximising use of prior test data and the potential for minimising animal use for future toxicity testing - the primary use of in silico toxicology methods in the pharmaceutical sciences are as decision support information. It is possible for in silico toxicology methods to complement and strengthen the evidence for certain regulatory review processes, and to enhance risk management by supporting a more informed decision regarding priority setting for additional toxicological testing in research and product development. There are also several challenges with these continually evolving methods which clearly must be considered. This mini-review describes in silico methods that have been researched as Critical Path Initiative toolkits for predicting toxicities early in drug development based on prior knowledge derived from preclinical and clinical data at the US Food and Drug Administration, Center for Drug Evaluation and Research.
\end{abstract}

Keywords: in silico toxicology, in silico methods, QSAR, SAR, computational toxicology, drug safety, genotoxicity, cardiac safety

\section{Introduction}

Born out of computational chemistry and chemoinformatics, in silico methods for toxicology testing have brought new insight into several areas of toxicology, including new predictive tools and datamining approaches to help make more effective use of large repositories of the results from in vitro and animal toxicology studies with xenobiotics. Substances that have been examined with these approaches include human pharmaceuticals, food ingredients, environmental agents and other chemicals to which there is significant human exposure. The use of in silico methods directed towards the evaluation of safety endpoints, as well as the deployment of chemoinformatics approaches in the analysis of genome responses after exposure to xenobiotics, has been supported by authorities through enacted legislation in the European Union-eg the Registration, Evaluation, Authorisation and Restriction of Chemicals (REACH) legislation-to help to reduce, refine and replace animal testing. ${ }^{1}$ In addition, recommendations recently made by the US National Research Council in its report, Toxicity Testing for the 21st Century: A Vision and a Strategy, also lend strong support to the use of computerbased technologies for the assessment of toxicities, with an emphasis on those relevant to protecting public health. ${ }^{2}$ The US Food and Drug Administration (FDA) recognises the need to develop and identify new product development and technical tools, for example, using computational, 
statistical, bioinformatics and genomics methods as part of its broad-based national strategy for transforming the way that FDA-regulated products are developed, evaluated and manufactured. ${ }^{3}$ The FDA's Critical Path Initiative describes this goal in its landmark report, Innovation/Stagnation: Challenge and Opportunity on the Critical Path to New Medical Products. ${ }^{4}$ Among other opportunities, the publication points out the potential to develop computer-based (in silico) methods as tools for the early detection of toxicity prior to significant human exposure to products that the agency regulates. ${ }^{4}$ Consistent with the aforementioned initiatives, the development and evaluation of in silico methods and databases in toxicology have been of interest to the FDA Center for Drug Evaluation and Research (CDER), Office of Pharmaceutical Science (OPS)..$^{5-11}$ In this mini-review, in silico toxicology models and databases at FDA/CDER/ OPS Science and Research Staff (SRS) will be described in their context as Critical Path Initiative toolkits.

\section{Goals and applied uses of in silico toxicology models}

One of the goals of the FDA Critical Path Initiative is to predict the toxicity of drugs early in their development, thus enabling the identification of safety signals prior to human exposure to, and significant investment in, new drug products. The in silico toxicology models that have been investigated at FDA/CDER/OPS primarily have been predictive tools. The principal approach has involved computational modelling of drug molecular structure under the (quantitative) structure-activity relationship ((Q)SAR) paradigm. The central axiom of (Q)SARs is that the activity of molecules is reflected in their structure, and this is quantifiable. Although structure-activity modelling is nothing new, there are specific examples of its success in early drug development. $^{12}$ Importantly, there are limitations-and many of these have been pointed out in recent in-depth reviews and commentaries. ${ }^{6,13-15}$
Without question, advancing computer technology facilitates high-end, and high-speed, analysis for dealing with the complexity of biological endpoints, using varying statistical algorithms to drive the many computational analyses. The FDA Critical Path Initiative of developing better evaluation tools and harnessing bioinformatics applies to innovation, science and technology to help to predict safety of drug products.

From the practical standpoint of industrial drug development, computational models like (Q)SARs and other approaches may support lead optimisation and identification of undesirable structural features in drugs, and evaluate metabolites and impurities (theoretical and known) in the discovery process. These attributes then may be removed by chemical modifications to reduce toxicity. ${ }^{16,17}$ The practical use of (Q)SAR models as instruments for regulators responsible for safety assessment of regulated products, however, is a much newer concept. Through applied regulatory research at the FDA OPS, insight into the application of (Q)SAR models, in terms of strengths and, importantly, limitations, has been learned over the past decade. ${ }^{6}$ At this time, there is no specific guidance document available from the FDA on in silico models or computational toxicology for safety assessment. The use of (Q)SARs to identify structural alerts for predicting genetic toxicity is stated under a draft guidance regarding recommended approaches to the safety assessment of genotoxic and carcinogenic impurities in drug substances and products. ${ }^{18}$ There are many questions that naturally could arise with respect to the applied use of (Q)SAR models, and this is being addressed at the FDA through regulatory research, committee review and, ultimately, public comment on the draft guidance. From this author's perspective, there are some reasonable questions that can be asked with respect to applying (Q)SAR models for predicting genetic toxicity for regulatory applications. These include:

- What specific endpoints in genetic toxicity are most appropriate to model for safety predictions?

- What are the standards that would define an evidentiary base of quality data and steps for transforming it into a computational model? 
- What assumptions in the model construction process are permissible?

- Are public data sufficient to model the toxicity and cover the chemical space of proprietary drugs?

- Is identification of a simple structural alert by an in silico screen sufficient, or are quantitative statistical probabilities needed?

- Must the model and computational algorithm used as the basis for the prediction be 100 per cent transparent?

- How does one deal with prediction conflicts, when one computational software program and model predicts one outcome, yet another computational platform predicts a different one?

- What are the factors and procedures to consider when a computational model is updated and leads to a different answer over time?

- How might consensus predictions be made, and are they really necessary?

- What level of predictivity (sensitivity and specificity) of a computational model is preferred, and under what use conditions?

- What methods should be employed that would be sufficient to establish the validation of a computational model before its regulatory application with human pharmaceuticals?

- Is there a preference for global multipurpose - One so called global models; local models constructed of highly smiliar chemicals; or more importantly, models based on high drug content?

- Is the current cadre of in silico toxicology tools reliable for assessing the risk of non-DNA-reactive drugs?

The answers to these questions and many others could lead to criteria that would be important in establishing the validity of (Q)SAR models for regulatory use in the USA. There are clearly questions outstanding with respect to the use of computational modelling approaches to predicting drug-related toxicity from a regulatory science standpoint. A recent article from the pharmaceutical industry echoes the questions regarding the suitability of public data for predicting the chemical space held by proprietary drugs, and the widespread use of evaluating predictive performance based on weak cross-validation strategies, as opposed to the more rigorous method of external validation. ${ }^{19}$ Cross-validation involves building an in silico toxicology model ((Q)SAR or similar), then removing a small part of the dataset (eg 10 per cent) and rebuilding the model to predict the toxicity endpoint being modelled (eg bacterial mutagenicity), using the test set that was removed. External validation is clearly more rigorous and representative of practical use of an in silico toxicology model. It involves testing the performance of the model using a set of test drugs that are foreign to the model and were never used in the construction of the in silico model. There are many such evaluations in the literature for genetic toxicity. $^{20,21}$

Despite the limitations and need for improving research techniques in computational modelling, the goals and applied uses of in silico toxicology models and databases remain unchanged. It is through rigorous research and validation that a better process for understanding the in silico approach will be identified.

\section{Critical Path Initiative toolkits}

The SRS at the FDA/CDER is located in the OPS immediate office. They conduct various types of research on current drug regulatory science issues. They support the development of regulatory guidance and policy for CDER, through the conduct of sound science in various areas of interest to the Center, such as nanotechnology, the evaluation of approaches to improving generic drug bioequivalence, the development of better regulatory and scientific tools for assessment of the environmental impact of drugs and the development of approaches for safety assessment of drugs. In silico toxicology models and databases are some of the FDA's Critical Path Initiative tools that the SRS uses to accomplish these goals, which may ultimately help to guide decision making and fulfil the SRS's regulatory responsibilities. Current activities in the SRS with respect to Critical Path Initiative tools in the 
Table I. In silico Critical Path Initiative toolkits at the FDA/CDER Office of Pharmaceutical Science, Science and Research Staff

\begin{tabular}{|l|l|}
\hline Drug safety issue & $\begin{array}{l}\text { Description of in silico } \\
\text { project }\end{array}$ \\
\hline QT prolongation & $\begin{array}{l}\text { Project: Building an in silico tool } \\
\text { for screening new drugs for QT } \\
\text { prolongation potential, using } \\
\text { human clinical trial data }\end{array}$ \\
\hline Phospholipidosis & $\begin{array}{l}\text { FDA/CDER working group on } \\
\text { drug-induced phospholipidosis. }\end{array}$ \\
\hline Database and development \\
of predictive computational \\
models of drug-induced \\
phospholipidosis
\end{tabular}

computational sciences are provided in Table 1. A brief outline of the toolkits being developed and evaluated in major projects follows.

\section{Computational modelling for QT prolongation and Torsades de points}

Torsades de points (TdP) is a potentially lethal cardiac arrhythmia that can be induced by several drugs. ${ }^{22}$ Lethal adverse events linked to TdP can halt a clinical trial and can be sufficient to terminate a drug's development. Thus, the clinical evaluation of a drug for TdP-related arrhythmia is a critical safety concern to protect public health and affects not only therapeutic cardiac drugs, but all new drugs with systemic exposure. QT interval prolongation is associated with $\mathrm{TdP}$ and measurement of QT interval effects is adopted into guidance by the FDA and international health authorities as a clinical evaluation method for assessing the proarrhythmia potential of new drugs. ${ }^{23,24}$ International Conference on Harmanisation ( $\mathrm{ICH})$ of Technical Requirements for Registration of Pharmaceuticals for Human use guidelines $\mathrm{ICH}$ S7B and ICH E14 delineate how QT/QTc interval prolongation for non-antiarrhythmic drugs can be addressed in preclinical studies and clinical evaluations of drugs under regulatory review and development. $^{25,26}$ In recognising the public health concern and the aforementioned guidance documents, a Critical Path Initiative project is in progress. This is aimed at developing validated in silico computational models for predicting clinical QT interval prolongation. The goal is to develop an in silico predictive tool for clinical evaluators, based on computational analysis of drug structureactivity relationships under the framework of the threshold of regulatory concern and other considerations related to a drug's safety profile. Importantly, the in silico models will be principally based on outcomes from gold standard agency-reviewed clinical trial data, such as thorough QT study (TQT) data. It is anticipated that such a model will add a higher level of quality compared with in silico models based on preclinical data. It is believed that better tools for predicting QT prolongation in qualitative (binary output) and quantitative (magnitude of QT prolongation) terms will be useful for directly supporting the risk assessment approaches used at the FDA/CDER during the drug development and regulatory evaluation of new drugs. Thus, an in silico predictive model, based on computational analysis of drug structure and the known potential of drugs to prolong the QT interval based on TQT clinical study outcomes, is being designed and 
developed using a hypothesis-based approach. This innovative work is being conducted in collaboration with clinical reviewers at CDER. It is hoped that a more important outcome from this research will be the development of the Critical Path toolkit for cardiac safety assessment, in terms of supporting regulatory safety evaluations for new drugs for their QT-proarrhythmia potential, to protect clinical trial subjects during drug development, as well as patients after drug marketing, from sudden lethal arrhythmias.

\section{Predictive modelling for drug-induced phospholipidosis}

Drug-induced phospholipidosis (PL) is the excessive accumulation of phospholipids and drug in cells, and has been observed in various tissue types, including lung, liver, kidney, heart, eye and brain. $^{27} \mathrm{PL}$ is a recurrent pathological feature in toxicity studies, and while it is more common in animals, it is also observed in humans. There are a large number of pharmacological classes of PL-inducing drugs, and this has been taken advantage of at the FDA/CDER in terms of applying chemical structure-based computational assessments, such as (Q)SARs. ${ }^{28}$ Moreover, most PL-inducing drugs are cationic amphiphilic drugs (CADS), and so there is evidence for a chemical structure-based explanation for why drugs induce PL. The FDA/CDER has built a database that contains hundreds of confirmed PL-positive drugs. This database is built upon both public (non-proprietary) and private (proprietary) study data derived from the literature, new drug applications and investigational new drug applications. ${ }^{29}$ Based on this database, the FDA/CDER has been able to construct in silico models to predict PL. ${ }^{28,29}$ This is an ongoing project and, as more new drugs are identified and confirmed to have PL-inducing effects, the model can be expanded and enhanced, so that the molecular coverage of the PL model will increase. The in silico PL models that the FDA/CDER is developing can thus be applied in risk management approaches to screening out PL-inducing drugs during development, and may aid regulators in assessing whether there should be a clinical concern when PL is found in animals. ${ }^{29}$

\section{In silico screening of botanical constituents for hepatic safety}

More than 50 million American women use menopausal hormone therapy (MHT) to manage menopausal symptoms. ${ }^{30}$ The public's concerns regarding the risks of MHT, however, have increased women's interest in alternatives such as botanical extracts, which have come under scrutiny for safety. Women's health research experts have reported that the most commonly used botanical agents in the USA for menopause-related symptoms are black cohosh, red clover, hops and chasteberry. ${ }^{31-33}$ Following case reports of liver injury in women who had consumed various types of botanical agents for MHT, a research project is being conducted for the FDA Office of Women's Health, using advanced in silico computational tools systematically to screen individual chemical constituents known to be present in these agents for hepatic safety signals. ${ }^{34-35}$ This research is also being conducted in collaboration with the CDER Botanical Drug Review team. Several computational approaches employing classic structure-activity relationships, probabilistic reasoning, machine learning methods, systems biological pathway analysis and chemo-informatics data mining are being deployed and combined with expert human knowledge to deliver toxicity and human adverse effect predictions on the chemicals identified in the botanical agents to produce a risk level and identify structural features associated with liver toxicity. The objective is to profile constituents that could be responsible for the hepatobiliary human adverse effects reported. Important safety signals are being identified through computational analysis and are reported to FDA regulatory groups at CDER and the Center for Applied Nutrition and Research (CFSAN) responsible for reviewing the safety of products containing botanical agents. In addition, the FDA Office of Women's Health is being provided with the results to support its research programme and initiatives. A large body of this work, which identified several structural features (such as phenolic, flavone, isoflavone and 4-hydroxyacetophenol structures) and botanical chemicals that may be of interest for hepatic safety, was recently published. ${ }^{36}$ Several 
botanical chemicals which were predicted to be positive for producing effects in the liver and were confirmed by animal toxicology data include protocatechuic acid, benzofuran and 4-vinylphenol. Estimated human exposure data were also provided in the study, to compare against the lowest level of these chemicals to produce an adverse effect and the level producing no adverse effect in animals. ${ }^{36}$ In the long term, this study will add scientific data to help to further the understanding of safety margins and to prioritise constituents and botanical mixtures for toxicology testing, and will provide insight into the current state of knowledge on the phytochemical content of these widely used botanical agents.

\section{Human-specific drug metabolites and P450 inhibition modelling}

The knowledge of a new drug's metabolic fate in humans is important in the discovery and development stages, as well as in FDA regulatory reviews. Because metabolic activation or off-target polypharmacology of drug metabolites can lead to undesirable and/or adverse effects in humans, predicting the human metabolic pathways of drugs in discovery, prior to the conduct of a clinical trial, can be important. Thus, use of in silico simulation of drug metabolism for the prediction of humanspecific drug metabolites and pharmacokinetics is another research focus for the SRS. This research has involved evaluating drug metabolism software for its ability qualitatively to predict the metabolites of drugs, as well as to simulate pharmacokinetic parameters and estimate bioavailability and the drug-metabolising enzymes responsible for producing the metabolites. Although characterisation of drug metabolism is submitted in sponsor submissions to the agency, and most clinically significant metabolites are measured in drug development programmes, there may still be questions that arise regarding which metabolites are specifically formed exclusively in humans and not in other species. Additionally, there are often questions about how data can be extrapolated from animals to humans, and how knowledge about metabolites that arise specifically in humans can help address why there may be differences between species, and how to make extrapolations. In exploratory research, the SRS is evaluating different in silico approaches and validating computational metabolic systems to determine the accuracy of predicting in vivo human drug metabolite structures. In a recent such study, a dataset of 17 hepatotoxic drugs was used as the base information for comparing the predictions with known human drug metabolites. ${ }^{37}$ It was found that computational metabolic software was able to predict 14 out of 23 major human metabolites from this dataset. ${ }^{37}$ It was also found that in silico technology was able to predict the putative toxic pathway (acylation) and known human metabolite of one of the drugs, whereas experimental data from rodent liver microsomal studies did not show evidence for this pathway. In addition, computational modelling involving (Q)SARs for cytochrome P450 (CYP) 2D6 and CYP3A4 enzyme inhibition has also been conducted as a modelling project, with the creation of validated models intended to aid the prediction of potential drugdrug interactions.

\section{Conclusion}

The FDA's Critical Path Initiative was launched in 2004 with the publication of a landmark report recognising that a) the decline in the number of new drugs approved in the USA is a public health concern; b) the rising costs of drug development is prohibitive; and c) there is a need to use scientific discoveries in biomedical science to modernise and develop safer and effective drugs. ${ }^{4}$ The aforementioned research in drug database building and in silico computational modelling fits with the core mission of the FDA Critical Path Initiative. These methodologies are intended to predict drug-related toxicity earlier and to do this with emerging chemo- and bioinformatics approaches, combined with knowledge from over a hundred years of animal toxicology study data that the agency has received and reviewed. This work is expected to lead to better evaluation tools in predictive science, especially for preclinical and clinical drug safety areas. The newest approach, which we expect to be the most enabling and to provide breakthroughs in 
this area, is the use of clinical trial data that the agency has reviewed to construct predictive computational models directed at helping to support risk assessments in cardiac safety and, ultimately, to lead to safer and effective medicines for public health.

\section{Acknowledgments}

The author reports no conflicts of interest. The author alone is responsible for the content and writing of the article. The content of this publication does not necessarily reflect the views or policies of the Department of Health and Human Services, nor does the mention of trade names, commercial products, or organisations imply endorsement by the US Government. This paper reflects the current thinking and experience of the author.

(C) Luis G. Valerio, Jr., 2011

\section{References}

1. EU (2006), 'Regulation (EC) No $1907 / 2006$ of The European Parliament and of the Council concerning the Registration, Evaluation, Authorisation and Restriction of Chemicals (REACH), establishing a European Chemicals Agency, amending Directive 1999/45/EC and repealing Council Regulation (EEC) No 793/93 and Commission Regulation (EC) No 1488/94, as well as Council Directive 76/769/EEC and Commission Directives 91/155/EEC, 93/67/EEC, 93/105/EC and 2000/21/EC', OJEU Vol. L396 (EC 1907/2006), pp. 1-843.

2. NRC (2007), Toxicity Testing in the 21st Century: A Vision and a Strategy, National Academy Press, Washington, DC.

3. FDA (2010), 'Critical Path Initiative', http://www.fda.gov/ ScienceResearch/SpecialTopics/CriticalPathInitiative/default.htm. US Department of Health and Human Services, Rockville, MD (accessed 15th June, 2010).

4. FDA (2004), 'Challenge and opportunity on the critical path to new medical products', http://www.fda.gov/ScienceResearch/SpecialTopics/ CriticalPathInitiative/CriticalPathOpportunitiesReports/ucm()77262.htm. US Department of Health and Human Services, US Food and Drug Administration, Rockville, MD.

5. Yang, C., Valerio, L.G., Jr. and Arvidson, K.B. (2009), 'Computational toxicology approaches at the US Food and Drug Administration', Altern. Lab. Anim. Vol. 37, pp. 523-531.

6. Valerio, L.G., Jr. (2009), 'In silico toxicology for the pharmaceutical sciences', Toxicol. Appl. Pharmacol. Vol. 241, pp. 356-370.

7. Valerio, L.G., Jr., Arvidson, K.B., Busta, E., Minnier, B.L. et al. (2010), 'Testing computational toxicology models with phytochemicals', Mol. Nutr. Food Res. Vol. 54, pp. 186-194.

8. Valerio, L.G., Jr. (2008), 'Tools for evidence-based toxicology: Computational-based strategies as a viable modality for decision support in chemical safety evaluation and risk assessment', Hum. Exp. Toxicol. Vol. 27, pp. 757-760.

9. Valerio, L.G., Jr., Yang, C., Arvidson, K.B. and Kruhlak, N.L. (2010), 'A structural feature-based computational approach for toxicology predictions', Expert Opin. Drug Metab. Toxicol. Vol. 6, pp. 505-518.

10. Valerio, L.G., Jr., Arvidson, K.B., Busta, E., Minnier, B.L. et al. (2010), 'Testing computational toxicology models with phytochemicals', Mol. Nutr. Food Res. Vol. 54, pp. 186-194.

11. Valerio, L.G., Jr. (2010), 'Computational science in drug metabolism and toxicology' [Editorial], Expert Opin. Drug Metab. Toxicol. Vol. 6, pp. $781-784$.
12. Evers, A. and Klebe, G. (2004), 'Successful virtual screening for a submicromolar antagonist of the neurokinin-1 receptor based on a ligandsupported homology model', J. Med. Chem. Vol. 47, pp. 5381-5392.

13. Combes, R.D. (2010), 'Is computational toxicology withering on the vine?', Arch. Toxicol. Vol. 84, pp. 333-336.

14. Johnson, S.R. (2007), 'The trouble with QSAR (or how I learned to stop worrying and embrace fallacy)', J. Chem. Inf. Model. Vol. 48, pp. 25-26.

15. Greene, N. and Naven, R. (2009), 'Early toxicity screening strategies', Curr. Opin. Drug Discov. Devel. Vol. 12, pp. 90-97.

16. Merlot, C. (2010), 'Computational toxicology - A tool for early safety evaluation', Drug Discov. Today Vol. 15, pp. 16-22.

17. Boyer, S. (2010), 'The use of computer models in pharmaceutical safety evaluation', Altern. Lab. Anim. Vol. 37, pp. 467-475.

18. FDA (2008), Draft guidance for industry: Genotoxic and carcinogenic impurities in drug substances and products: Recommended approaches, US FDA/CDER, US Department of Health and Human Services, Silver Spring, MD.

19. Naven, R.T., Louise-May, S. and Greene, N. (2010), 'The computational prediction of genotoxicity', Expert Opin. Drug Metab. Toxicol. Vol. 6, pp. 797-807.

20. Benigni, R., Bossa, C., Tcheremenskaia, O. and Giuliani, A. (2010), 'Alternatives to the carcinogenicity bioassay: In silico methods, and the in vitro and in vivo mutagenicity assays', Expert Opin. Drug Metab. Toxicol. Vol. 6, pp. 809-819.

21. Benigni, R. and Bossa, C. (2008), 'Predictivity and reliability of QSAR models: The case of mutagens and carcinogens', Toxicol. Mech. Methods Vol. 18, pp. 137-147.

22. Lee, N., Authier, S., Pugsley, M.K. and Curtis, M.J. (2010), 'The continuing evolution of torsades de pointes liability testing methods: Is there an end in sight?', Toxicol. Appl. Pharmacol. Vol. 243, pp. 146-153.

23. Roden, D.M. (2004), 'Drug-induced prolongation of the QT interval', N. Engl. J. Med. Vol. 350, pp. 1013-1022.

24. Piccini, J.P., Whellan, D.J., Berridge, B.R., Finkle, J.K. et al. (2009), 'Current challenges in the evaluation of cardiac safety during drug development: Translational medicine meets the Critical Path Initiative', Am. Heart J. Vol. 158, pp. 317-326.

25. FDA (2005), International Conference on Harmonisation: Guidance on E14 clinical evaluation of QT/QTc interval prolongation and proarrhythmic potential for non-antiarrhythmic drugs; availability. Notice. Department of Health and Human Services Vol. 70, pp. 61134-61135.

26. Cavero, I. and Crumb, W. (2005), 'ICH S7B draft guideline on the nonclinical strategy for testing delayed cardiac repolarisation risk of drugs: A critical analysis', Expert Opin. Drug Saf. Vol. 4, pp. 509-530.

27. Cartwright, M.E., Petruska, J., Arezzo, J., Frank, D. et al. (2009), 'Phospholipidosis in neurons caused by posaconazole, without evidence for functional neurologic effects', Toxicol. Pathol. Vol. 37, pp. 902-910.

28. Kruhlak, N.L., Choi, S.S., Contrera, J.F, Weaver, J.L. et al. (2008), 'Development of a phospholipidosis database and predictive quantitative structure-activity relationship (QSAR) models', Toxicol. Mech. Methods Vol. 18, pp. 217-227.

29. FDA (2010), 'The regulatory challenges of drug-induced phospholipidosis', http://www.fda.gov/downloads/AdvisoryCommittees/Committees MeetingMaterials/Drugs/AdvisoryCommitteeforPharmaceuticalScienceand ClinicalPharmacology/UCM210798.pdf. Department of Health and Human Services, Bethesda, MD (accessed 14th June, 2010).

30. NAMS. The North American Menopause Society web page (2010), 'Hormone therapy statistics', http://www.menopause.org/hormonetherapystats.aspx. NAMS, Mayfield Heights, OH (accessed 15th May, 2010).

31. Kurzer, M.S. and Xu, X. (1997), 'Dietary phytoestrogens', Ann. Rev. Nutr. Vol. 17, pp. 353-381.

32. Liu, J., Burdette, J.E., Xu, H., Gu, C. et al. (2001), 'Evaluation of estrogenic activity of plant extracts for the potential treatment of menopausal symptoms', J. Agric. Food Chem. Vol. 49, pp. 2472-2479.

33. Lieberman, S. (1998), 'A review of the effectiveness of Cimicifuga racemosa (black cohosh) for the symptoms of menopause', J. Women's Health Vol. 7, pp. 525-529. 
34. Mahady, G.B., Dog, T.L., Barrett, M.L., Chavez, M.L. et al. (2008), 'United States Pharmacopeia review of the black cohosh case reports of hepatotoxicity', Menopause Vol. 15, pp. 628-638.

35. Betz, J., Anderson, L., Avigan, M.I., Barnes, J. et al. (2009), 'Black cohosh. Considerations of safety and benefit', Nutr. Today Vol. 44, pp. $155-162$.
36. Wang, W.J., Dou, J., Cross, K.P. and Valerio, L.G., Jr. (2011), 'Computational analysis for hepatic safety signals of constituents present in botanical extracts widely used by women in the United States for treatment of menopausal symptoms', Reg. Toxicol. Pharmacol. Vol. 59, pp. 111-124.

37. Valerio, L.G., Jr. (2010), 'The in silico prediction of human-specific metabolites from hepatotoxic drugs', Curr. Drug Discov. Technol. Vol. 7, pp. 170-187. 